\title{
LA TÉCNICA DEL ORIGAMI PARA LA PRODUCCIÓN DE TEXTOS DE NIÑOS DEL SEGUNDO GRADO DE PRIMARIA DEL CENTRO EDUCATIVO DE MENORES N 30225 “LA ALBORADA" DE EL TAMBO, HUANCAYO
}

\begin{abstract}
THE ORIGAMI TECHNIQUE TO PRODUCE TEXTS FOR CHILDREN OF SECOND GRADE PRIMARY AT THE CENTER EDUCATIONAL OF CHILDREN N 30225 “LA ALBORADA” IN THE TAMBO, HUANCAYO
\end{abstract}

Andrés Carhuamanta LLanco', Rosa Ercira Díaz Rojas'

\section{RESUMEN}

La investigación tuvo el propósito de contribuir en mejorar la producción de textos de los niños del segundo grado de Primaria del Centro Educativo de Menores N ${ }^{\circ} 30225$ "La Alborada" de El Tambo, Huancayo, a través de la técnica del origami. La producción de textos contribuye a la formación del pensamiento organizado, para la comunicación y apoyo a la construcción del conocimiento del niño del segundo grado de educación primaria. Un niño que no produce textos tendrá dificultades en su aprendizaje posterior; no podrá comunicar sus ideas, intereses, necesidades, sentimientos, etc. La investigación fue cuasiexperimental de: "dos grupos no equivalentes con preprueba y postprueba". La muestra fue de carácter intencional; constituido por 46 niños del segundo grado. La sección A con 23 niños conformó el grupo control y la sección B con 23 niños, el grupo experimental. Se concluyó que la aplicación de la técnica del origami muestra alto grado de eficacia en la producción de textos en niños del segundo grado de primaria; debido a que la chi calculada es mayor que la chi de tabla $(32,124$ $>5,991$ ).

Palabras clave: Origami, niños, educación primaria, textos.

\begin{abstract}
The investigation had the purpose of contributing in improving the production children of the primary second grade children texts of the Educational Center of Children N 30225 "La Alborada" of El Tambo, Huancayo, through the technique of the origami. The production of texts contributes to the formation of the organized thought, for communication and supports the construction of knowledge of the child of the second grade of primary education. A child that does not produce texts will have difficulties in his later learning; he/she will not be able to communicate their ideas, interests, necessities, feelings, etc. The investigation was cuasiexperimental of: "two non equivalent groups with pre test and post test". The sample was of intentional character; constituted by 46 children of the second grade. The section A with 23 children it conformed the group control and the section B with 23 children, the experimental group. It was concluded that the application of the technique of origami shows high grade of effectiveness in the production of texts in children of the second grade of primary; because the calculated chi is bigger than the chart chi $(32,124>$ 5,991).
\end{abstract}

Key words: Origami, children, primary education, texts. 


\section{INTRODUCCIÓN}

Los estudios actuales de la lingüística demuestran que cuando hablamos, construimos textos, pero la construcción y producción de textos escritos es un proceso de mayor complejidad, porque requiere que aquello que se va a transmitir o comunicar se transforme a un código de segundo nivel de abstracción o simbolización, como es la escritura, tomando en cuenta sus características lingüísticas, (1) significativamente la calidad y la equidad del aprendizaje de la lectura y la escritura de los niños entre 5 y 9 años; concluyen que: los niños son capaces de producir distintos tipos de textos según su propósito y características lingüísticas.

La investigación tuvo como objetivo probar experimentalmente el grado de eficacia de la técnica del origami en la producción de textos en niños; ya que ello permite realizar composiciones estilizadas de animales, flores, objetos, aviones, etc; cuya característica fundamental es la tridimensionalidad; permitiendo en primera instancia realizar una composición plástica con las figuras de origami, luego, en base a ella, producir textos escritos. Adicionalmente, el origami, también ayuda a desarrollar en el niño las habilidades motoras finas y gruesas; la memoria, la capacidad creativa y la sensibilidad humana entre otros.

\section{MATERIAL Y MÉTODOS}

Se utilizó el diseño cuasiexperimental de: "Dos grupos no equivalentes con preprueba y postprueba".

La población fue conformada por 240 niños del segundo grado de primaria. La muestra fue no probabilística de manera intencional; constituido por 46 niños La sección A con 23 niños fue el grupo control y la sección B con 23 niños fue el grupo experimental.

Al grupo experimental se aplicó el tratamiento, mientras que el grupo control con el programa normal diseñado por la profesora de aula.

Para recolectar los datos, se utilizaron las técnicas: fichaje y la observación directa. El instrumento para recabar la información fue la lista de cotejo de conductas para la producción de textos. Dicho instrumento sirvió para la prueba de entrada y de salida de ambos grupos.
Los indicadores del instrumento de evaluación fueron: Demuestra dominio de las características del mensaje escrito (claridad, coherencia, concisión, precisión, variedad; presenta con secuencia lógica los hechos; en sus textos manuscritos, la escritura es legible; utiliza adecuadamente la puntuación (puntos, comas, guiones, etc.); las letras mayúsculas son utilizadas de acuerdo a las normas; en sus textos manuscritos hay regularidad de la letra. Como elementos de valoración (A) logro, (B) en proceso, (C) en inicio.

Para probar las hipótesis, se aplicó la prueba de chi cuadrada. Como materiales: círculos de papel de lustre de diferentes colores y tamaños, papel bond de 75 gramos, tijera, cola sintética, láminas motivadoras de origami y modelos de producción de textos, como cartas, afiches, avisos, notas, etc.

\section{RESULTADOS}

En la Tabla 1, se observa que todos los indicadores de la chi calculada, es mayor que la chi de tabla; el

Tabla 1. Comprabación estadística por indicadores

\begin{tabular}{|c|c|c|c|}
\hline Grupo & Indicadores & $X^{2}$ calculada & $\mathrm{X}^{2}$ tahla \\
\hline \multirow{6}{*}{$\begin{array}{c}\text { Cont'ol } \\
\text { Experimental }\end{array}$} & Indic ador 1 & $\bar{i}, 098$ & 5,931 \\
\hline & Indic ador 2 & 3,69 & 5,931 \\
\hline & Indic ador 's & $b, y$ & $5, y=11$ \\
\hline & Indic ador 4 & 6,244 & 5,931 \\
\hline & Illudil:adur 5 & 6,134 & 5.931 \\
\hline & Indic ador 6 & 6 & 5.931 \\
\hline
\end{tabular}

indicador 1. Dominio de las características del mensaje escrito, la chi calculada es más alta que en los demás indicadores; mientras que en el indicador 6 . En sus textos manuscritos hay regularidad de la letra, es la más baja.
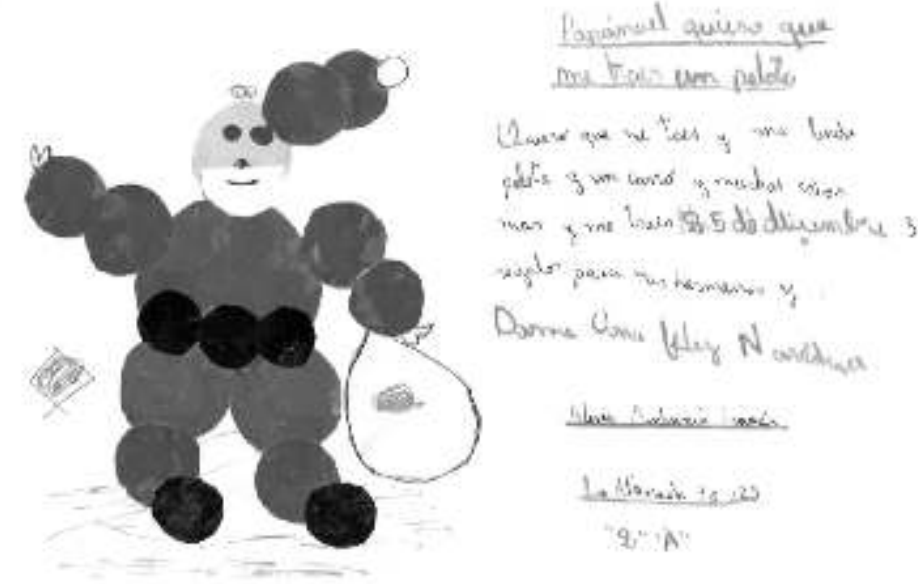
En la tabla 2, se aplicó la chi cuadrada a las respuestas de las pruebas de salida de ambos grupos y en forma general.

Tahla 2. Resultados de la C.hi C.uadrada en general de las pruphas de salida en aubos grupos

\begin{tabular}{lccc}
\hline Grupo & Prueba & $\boldsymbol{x}^{2}$ calculada & $\boldsymbol{x}^{2}$ tabla \\
\hline $\begin{array}{l}\text { Controly } \\
\text { Experimental }\end{array}$ & Salida & 32,124 & 5,991 \\
\hline
\end{tabular}

\section{DISCUSION}

En la prueba de salida obtuvo un valor de la chi calculada de 32,124 a diferencia de la chi de tabla de $5,991(32,124>5,991)$ por lo que se rechaza la hipótesis nula y se acepta la hipótesis alterna. Esto significa que la proporción de calificativos A, B y C de la producción de textos en la prueba de salida es significativamente diferente entre el grupo control y el grupo experimental, notándose que del grupo experimental ganan en respuestas en el calificativo $A$, en tanto el grupo control tienen más respuestas en $B$.

Al aceptar la hipótesis alterna, se está demostrando que la técnica del origami influye significativamente en la producción de textos en niños del segundo grado de primaria.

En la prueba de salida, la chi calculada fue mayor que la chi de tabla $(32,124>5,991)$; puesto que con ella se demuestra que los alumnos mejoraron su producción de textos; al demostrar dominio de las características del mensaje escrito (claridad, coherencia, concisión, precisión, variedad); presentando con secuencia lógica los hechos. En sus textos manuscritos, la escritura fue legible; y para su edad utilizaron adecuadamente la puntuación (puntos, comas, guiones, etc.); las letras mayúsculas fueron utilizadas de acuerdo a las normas; $y$ en sus textos manuscritos demostraron regularidad de la letra.

Comparando la presente investigación con la de, (1), podemos resaltar que tanto ellas como nosotros arribamos a resultados significativos. En nuestro caso, la variable independiente fue la técnica del origami; mientras que ellas utilizaron diferentes estrategias activas acompañadas con pequeñas historias y cuentos.

En la producción de textos por los niños, las investigadoras chilenas, ( 1 y 5 ), partieron de un trabajo individual y grupal, en el marco de proyectos de manera libre; mientras que nosotros partimos de una composición artística, libre y creativa con figuras de origami, y en base a ella, los niños produjeron textos libres y creativos. Esta estrategia metodológica obedeció a que la mayoría de los niños en nuestro medio sienten cierto temor o desidia por escribir ellos mismos un texto.

Teniendo en cuenta que la producción de textos es tan amplia y compleja para los niños, se hace necesario seleccionar y crear estrategias adecuadas para desarrollarlas; en ese sentido, la técnica del origami es eficaz para este propósito. Sin embargo, el docente tiene que avanzar de lo simple a lo complejo, de lo fácil o lo difícil, tratando de no cansar al alumno al realizar sus composiciones.

En base a las composiciones de origami que realizan los niños, producen diferentes textos: textos funcionales del área: lista de materiales, planificación de proyectos, Textos de comunicación como: afiches, recetas, cartas, etc.; y textos de expresión literaria: poemas, cuentos, refranes, adivinanzas y rimas.

\section{REFERENCIAS BIBLIOGRÁFICAS}

1. Jolibert J, et al. Interrogar y producir textos auténticos: Vivencias en el aula. Santiago de Chile: Edit. Dolmen Ediciones S.A;1998.

2. Aytüre Z. Nuevas ideas de origami. La Coruña: Edit. Everest S.A.; 1994.

3. Breda V. Origami. El arte del papel plegado. Buenos Aires: Edit. Kapelusz; 1972.

4. Hidalgo M. Pedagogía de la expresión escrita y la creatividad infantil. Lima: Edit. San Marcos; 2000.

5. Inostroza G, Jolibert J. Aprender a formar niños lectores y escritores. Chile: Dolmen Ediciones S.A.; 1997.

6. Kasahara K. Papiroflexia creativa. Madrid: Edit. Edaf S. A.; 1993.

7. Kneissler I. Origami, técnica japonesa de la plegadura del papel. París: Edic. Bouret; 1971.

8. Kneissler I. Origami Creativo. Barcelona: Edic. CEAC; 1992.

9. Krooshoop J. Felicitaciones con Origami. Barcelona: Edic. CEAC; 1993.

10. Lareo G. Manual Práctico de Cocotología y Papirología. Buenos Aires: Edit. El Ateneo; 1991.

11. Valladares,O. Comunicación integral. Lima: Edit. Mantaro; 2000.

E-mail:rosaercira@hotmail.com 